The International Journal of Engineering and Science (IJES)

|| Volume || 6 || Issue || 4 || Pages || PP 01-07 || 2017 ||

ISSN (e): $2319-1813$ ISSN (p): $2319-1805$

THE IJES

\title{
Finite Element Method Linear Rectangular Element for Solving Finite Nanowire Superlattice Quantum Dot Structures GaAs/AlGaAs
}

\author{
${ }^{1}$ Eman Ali Hussain, ${ }^{2}$ Jamil A. Al-Hawasy and ${ }^{3}$ Lamyaa H. Ali \\ ${ }^{1}$ Asst.Prof., Dr., Department of Mathematics, college of Science, University of AL-Mustansiriyah, Iraq \\ ${ }^{2}$ Asst.Prof., Dr., Department of Mathematics, college of Science, University of AL-Mustansiriyah, Iraq \\ ${ }^{3}$ Lec., Department of Mathematics, college of Science, University of AL-Mustansiriyah, Iraq
}

\begin{abstract}
-
This paper concerned with the solution of finite nanowire superlattice quantum dot structures GaAs/AlGaAs with a cylindrical cross-section determine by electronic states in various type of layers in terms of wave functions between structures containing the same number of barriers and wells (asymmetrical) or containing a different number (symmetrical). The solution is considered with the Finite element method with different base linear rectangular element to solve the one electron Ben Daniel-Duke equation. The results of numerical examples are compared for accuracy and efficiency with the finite difference method of this method and finite element method of linear triangular element. This comparison shows that good results of numerical examples.
\end{abstract}

Date of Submission: 17 February 2017 Date of Accepted: 07 April 2017

\section{INTRODUCTION}

The modeling of the electron states in semiconductor nanostructures remains a difficult computational task. In 1966, Ben Daniel and Duke, [1] are used space-charge effects on electron tunneling of the one electron model is formulated to describe Ben Daniel-Duke equation. There are various methods to solve this type of problems such as in 1988 Ghatak et. al., [2] used transfer matrix method. In 1990, Paasch et. al., [3] used envelope equation and wave function matching for narrow-gap semiconductors. In 1995, Mathine et. al., [4] applied computational Fourier series solution of the BenDaniel-Duke Hamiltonian for arbitrary shaped quantum wells. In 2003, Melnik et al, [5] applied finite element analysis of Nanowire superlattice structures. Whereas 2004, Melnik et al, [6] using finite difference method in cylindrical polar coordinates Nanowire superlattice. In 2016, Deyasi et. al., [7] applied numerically computed in presence of electric field using propagation matrix method. In this paper we propose a Finite element method and using different base linear rectangular element for solving Ben Daniel-Duke equation. This solution is considered with the solution of finite nanowire superlattice quantum dot structures GaAs/AlGaAs of a cylindrical cross-section and determine by electronic state in the two of layers GaAs (the well) and AlGaAs (the barrier), three of layers with well layers at both ends and six of layers with well layers at one end and barrier layers at the other end, in terms of wave functions, between structures containing the same number of barriers and wells (asymmetrical) and structures where the number of barrier layers is one above the number of well layers (symmetrical).

\section{DESCRIPTION OF THE BEN DANIEL-DUKE EQUATION[5],[6]}

The Nanowire superlattice (NWSL) is modeled as an ideal cylinder with sharp modulations in the longitudinal (or $z$-direction). The theory of the electron state used here is the one-band envelope function theory. In the application, the effective mass of the electron is dependent on the position, but does not depend on energy. The electronic structure can be found in general based on a multiband model for the envelope function:

$$
H \psi=E \psi,
$$

where $H$ is the Hamiltonian, $\psi$ is called eigenfunction, and $E$ is called energy eigenvalue.

Quantum mechanical problems in semiconductor heterostructures are often treated using a single band effective mass equivalent Hamiltonian. This means in a more general that only envelope functions as solutions of the equivalent Ben Daniel-Duke equation:

$$
-\frac{\hbar^{2}}{2} \nabla \cdot\left[\frac{1}{m(r)} \nabla \psi(r)\right]+V(r) \psi(r)=E \psi(r)
$$


where $m(r)$ is the effective mass in each superlattice layer, $\psi(r)$ is the wave function, $V(r)$ is the potential experienced by electron it can taken to be infinite outside the wire (vacuum) and piecewise inside, and $E$ is the energy.

The NWSL is modeled as a cylinder of infinite length, with alternating layers of GaAs (the well) and AlGaAs (the barrier) see Fig.(1). The NWSL's currently being grown are free standing. Eq. (2) is supplemented by Dirichlet boundary conditions (on the surface of the nanowire structures) which correspond to the case where the nanostructure environment is vacuum.

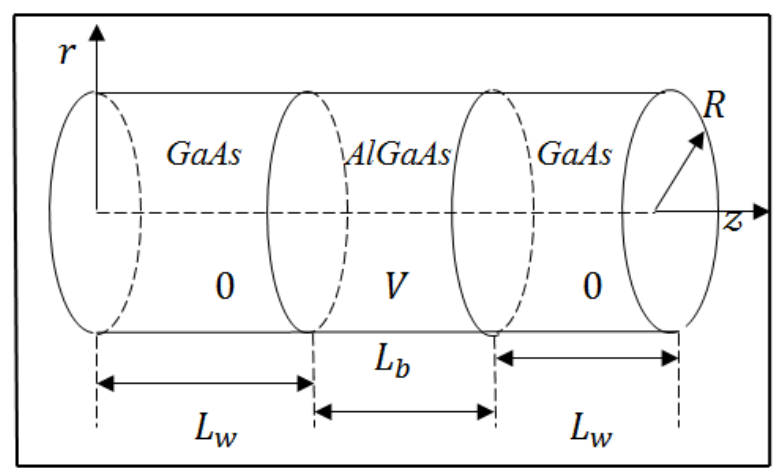

(a)

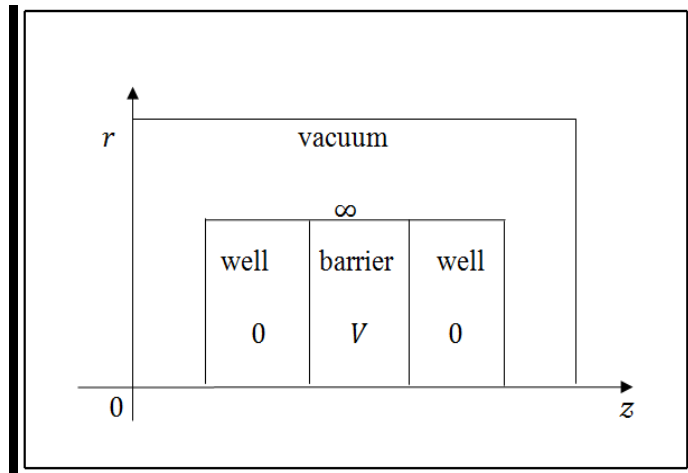

(b)

Fig. (1) (a)Nanowire superlattice model for a three layers, with well layers at both ends and (b) The domain for a unit cell of nanowire superlattice.

In this work we study a finite sequence of wells and barriers, with infinite potential barriers at the ends, three patterns are possible:

1. The ends are both wells,

2. At one end there is a well and at the other there is a barrier,

3. The ends are both barriers.

The structure in the first and third case is symmetric with respect to a center plane, and the wavefunctions are symmetric or antisymmetric. While the structure is asymmetric in the in the second case with respect to a center plane.

\section{FINITE ELEMENT SOLUTION}

To solve the one-electron Ben Daniel-Duke equation we write the gradient and the divergence operators in cylindrical coordinates, i.e.

$$
\frac{-\hbar^{2}}{2}\left[\frac{1}{r} \frac{1}{m} \frac{\partial \psi}{\partial r}+\frac{\partial}{\partial r}\left(\frac{1}{m} \frac{\partial \psi}{\partial r}\right)+\frac{1}{r} \frac{\partial}{\partial \phi}\left(\frac{1}{m} \frac{1}{r} \frac{\partial \psi}{\partial \phi}\right)+\frac{\partial}{\partial z}\left(\frac{1}{m} \frac{\partial \psi}{\partial z}\right)\right]+V \psi=E \psi
$$

Since the cylindrical is symmetry, $V=(r, z), m=(r, z)$, and $\psi=u(r, z) e^{i L}{ }_{x}$ where $L$ is an integer. The Ben Daniel-Duke equation, Eq. (3), becomes

$$
\frac{-\hbar^{2}}{2}\left[\frac{1}{r} \frac{\partial}{\partial r}\left(\frac{r}{m} \frac{\partial u}{\partial r}\right)-\frac{L^{2}}{m r^{2}} u+\frac{\partial}{\partial z}\left(\frac{1}{m} \frac{\partial u}{\partial z}\right)\right]+V u=E u \text { in } \Omega
$$

with the boundary condition

$$
\frac{\partial u}{\partial z}=0 \text {, in } \Gamma
$$

where $\Omega$ is a bounded open domain in the plane $\mathbb{R}^{2}$ with boundary $\Gamma$.

The Ben Daniel-Duke equation governing physical process in a cylindrical Nanowire superlattice model are described in terms of cylindrical coordinates. When the geometry, loading, and boundary conditions are independent of the circumferential direction ( $\phi$-coordinate in Fig. (1)), the problem is said to be axisymmetric and the Ben Daniel-Duke equation becomes in two-dimensional representing by $r$ and $z$.

\subsection{Weak Form}

Assume that $\Omega$ is a typical element, whether rectangular or quadrilateral, of the finite element mesh, and we develop the finite element model of (4) over $\Omega$. Various two-dimensional elements will be discussed in the sequel.

There are three steps in the development of the weak form of (4) over the typical element $\Omega$. 
The first step is formulated (4) by the following weak form

$$
0=\int_{\Omega}\left[\frac{-\hbar^{2}}{2}\left[w \frac{1}{m} \frac{\partial u}{\partial r}+w r \frac{\partial F_{1}}{\partial r}-\frac{w L^{2}}{m r} u+w r \frac{\partial F_{2}}{\partial z}\right]+w r V u-w r E u\right] d r d z
$$

Where

$$
F_{1}=\left(\frac{1}{m} \frac{\partial u}{\partial r}\right) \text { and } F_{2}=\left(\frac{1}{m} \frac{\partial u}{\partial z}\right)
$$

In the second step we distribute the differentiation among $u$ and $w$ equally. To achieve this, we integrate the first terms in $(4 \mathrm{a})$ by parts. We note the identities

$$
\begin{aligned}
& -w r \frac{\partial F_{1}}{\partial r}=r \frac{\partial w}{\partial r} F_{1}-r \frac{\partial}{\partial r}\left(w F_{1}\right) \\
& -w r \frac{\partial F_{2}}{\partial z}=r \frac{\partial w}{\partial z} F_{2}-r \frac{\partial}{\partial z}\left(w F_{2}\right)
\end{aligned}
$$

Next, we use the component form of the gradient (or divergence) theorem on the boundary $\Gamma$ and $d s$ using (6), and (7) in (5), we obtain

$$
\begin{aligned}
0=\int_{\Omega}\left[\frac{-\hbar^{2}}{2 m} w \frac{\partial u}{\partial r}\right. & \left.+\frac{\hbar^{2}}{2} r \frac{\partial w}{\partial r}\left(\frac{1}{m} \frac{\partial u}{\partial r}\right)+\frac{\hbar^{2} L^{2}}{2 m r} w u+\frac{\hbar^{2}}{2} r \frac{\partial w}{\partial z}\left(\frac{1}{m} \frac{\partial u}{\partial z}\right)+w r V u-w r E u\right] d r d z \\
& -\oint_{\Gamma} w\left[n_{1}\left(\frac{r}{m} \frac{\partial u}{\partial r}\right)+n_{2}\left(\frac{1}{m} \frac{\partial u}{\partial z}\right)\right] d s
\end{aligned}
$$

where $\vec{n}=\left(n_{1}, n_{2}\right)$ is an outer unite vector normal on $\Gamma$ and $d s$ is the length of an infinitesimal line element along the boundary.

From an inspection of the boundary integral in (8), we note that the specification of $u$ constitutes the essential boundary condition, and hence $u$ is the primary variable. The specification of the coefficient of the weight function in the boundary expression, i.e. we can let

$$
b_{n} \equiv n_{1}\left(\frac{r}{m} \frac{\partial u}{\partial r}\right)+n_{2}\left(\frac{1}{m} \frac{\partial u}{\partial z}\right)
$$

The third and last step of the formulation is to substitute the definition (9) in (8) and write the weak form of (4) as

$$
\begin{aligned}
0=\int_{\Omega}\left[\frac{-\hbar^{2}}{2 m} w \frac{\partial u}{\partial r}\right. & \left.+\frac{\hbar^{2}}{2} r \frac{\partial w}{\partial r}\left(\frac{1}{m} \frac{\partial u}{\partial r}\right)+\frac{\hbar^{2} L^{2}}{2 m r} w u+\frac{\hbar^{2}}{2} r \frac{\partial w}{\partial z}\left(\frac{1}{m} \frac{\partial u}{\partial z}\right)+w r V u-w r E u\right] d r d z \\
& -\oint_{\Gamma} w b_{n} d s
\end{aligned}
$$

\subsection{Finite Element Model of Ben Daniel-Duke equation}

Suppose that $u(r, z)$ is approximated over a typical finite element $\Omega$ by the expression

$$
u(r, z) \approx u_{h}^{e}(r, z)=\sum_{j=1}^{n} u_{j}^{e} \psi_{j}^{e}(r, z)
$$

where $u_{j}^{e}$ is the value of $u_{h}^{e}$ at the $j^{\text {th }}$ node $\left(r_{j}, z_{j}\right)$ of the element and $\psi_{j}^{e}$ are the Lagrange interpolation functions.

Substituting the finite element approximation (11) for $u$ into the weak form (10), we get

$$
\begin{gathered}
0=\int_{\Omega}\left[\frac{-\hbar^{2}}{2 m} w \sum_{j=1}^{n} u_{j}^{e} \frac{\partial \psi_{j}^{e}}{\partial r}+\frac{\hbar^{2}}{2} r \frac{\partial w}{\partial r}\left(\frac{1}{m} \sum_{j=1}^{n} u_{j}^{e} \frac{\partial \psi_{j}^{e}}{\partial r}\right)+\frac{\hbar^{2} L^{2}}{2 m r} w \sum_{j=1}^{n} u_{j}^{e} \psi_{j}^{e}+\frac{\hbar^{2}}{2} r \frac{\partial w}{\partial z}\left(\frac{1}{m} \sum_{j=1}^{n} u_{j}^{e} \frac{\partial \psi_{j}^{e}}{\partial z}\right)\right. \\
\left.+w r V \sum_{j=1}^{n} u_{j}^{e} \psi_{j}^{e}-w r E \sum_{j=1}^{n} u_{j}^{e} \psi_{j}^{e}\right] d r d z-\oint_{\Gamma} w d_{n} d s
\end{gathered}
$$

This equation must hold for every admissible choice of weight function $w$. Since we need $n$ independent algebraic equations to solve for the $n$ unknowns, $u_{1}^{e}, u_{2, \ldots}^{e}, u_{n,}^{e}$ we choose $n$ linearly independent functions for $w: w=\psi_{1}^{\oplus}, \psi_{2}^{\oplus}, \ldots, \psi_{n}^{\ominus}$. For each choice of $w$ we obtain an algebraic relation among $\left(u_{1}^{\oplus}, u_{2}^{\ominus}, \ldots, u_{n}^{\ominus}\right)$. We label the algebraic equation resulting from substitution of $w=\psi_{i}^{E}$ into (12): 


$$
\begin{aligned}
& 0=\sum_{j=1}^{n}\left\{\int _ { \Omega } \left[\frac{-\hbar^{2}}{2 m} \psi_{i}^{\theta} \frac{\partial \psi_{j}^{\varphi}}{\partial r}+\frac{\hbar^{2}}{2} r \frac{\partial \psi_{i}^{\varphi}}{\partial r}\left(\frac{1}{m} \frac{\partial \psi_{j}^{\varphi}}{\partial r}\right)+\frac{\hbar^{2} L^{2}}{2 m r} \psi_{i}^{\theta} \psi_{j}^{\theta}+\frac{\hbar^{2}}{2} r \frac{\partial \psi_{i}^{e}}{\partial z}\left(\frac{1}{m} \frac{\partial \psi_{j}^{\varphi}}{\partial z}\right)+r V \psi_{i}^{\theta} \psi_{j}^{\varphi}\right.\right. \\
& \left.\left.-r E \psi_{i}^{\varphi} \psi_{j}^{e}\right] d r d z\right\} u_{j}^{e}-\oint_{\Gamma} w d_{n} d s
\end{aligned}
$$

Problem (13) is supplemented by Neumann's boundary conditions if $L=0$. In the following subsections, we will solve the above meant form of the Ben Daniel-Duke equation by FEM using different base linear rectangular element and also in the next subsection we will solve it using FEM with linear rectangular element.

\subsection{Linear Rectangular Elements}

A solution of the weak form of Ben Daniel-Duke equation (10) and finite element matrices in (12) shows that $\psi_{j}^{\varphi}$ should be at least four linearly independent term and is linear in $r$ and $z$, with a bilinear term in $r$ and $z$ in $\Omega$ is of the form

$$
u_{1}^{\varphi}(r, z)=c_{1}^{\oplus}+c_{2}^{\varphi} r+c_{a}^{\varphi} z+c_{4}^{\oplus r z}
$$

This polynomial requires an element with four nodes. The linear rectangular element is a compatible element because on any side $u_{R}^{\ell}$ varies only linearly and there are two nodes to uniquely define it.

Here consider an approximation of the form (14) and use a rectangular element with sides $h$ and $l$. For the sake of convenience, choose a local cylindrical polar coordinate system $(\bar{r}, \bar{z})$ to derive the interpolation functions. Assume that

$$
u_{h}(\bar{r}, \bar{z})=c_{1}+c_{2} \bar{r}+c_{a} \bar{z}+c_{4} \bar{r} \bar{z}
$$

And require

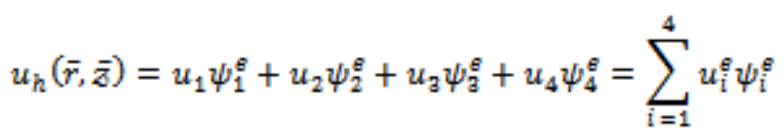

where

$$
\begin{aligned}
\psi_{1}^{e} & =\left(1-\frac{\bar{r}}{a}\right)\left(1-\frac{\bar{z}}{b}\right) . \\
\psi_{2}^{e} & =\frac{\bar{r}}{a}\left(1-\frac{\bar{z}}{b}\right), \\
\psi_{a}^{e} & =\frac{\bar{r}}{a} \bar{z}^{x} \\
\psi_{4}^{e} & =\left(1-\frac{\bar{r}}{a}\right) \frac{\bar{z}}{b^{x}}
\end{aligned}
$$

or, in concise form,

$$
\psi_{i}^{e}(\bar{r}, \bar{z})=(-1)^{i+1}\left(1-\frac{\bar{r}+\overline{r_{i}}}{a}\right)\left(1-\frac{\bar{z}+\bar{z}_{i}}{b}\right)
$$

where $\left(\bar{r}_{\mathrm{i}}, \bar{z}_{\mathrm{i}}\right)$ are the $(\bar{r}, \bar{z})$ cylindrical polar coordinate of node $i$. The interpolation function $\psi_{\mathrm{i}}^{\oplus}$ have the properties

$$
\psi_{i}^{g}\left(\bar{r}_{j}^{g}, \bar{z}_{j}^{g}\right)=\delta_{i j} \quad(i, j=1, \ldots, 4), \quad \sum_{i=1}^{4} \psi_{i}^{e}=1
$$

Now, to solve Ben Daniel-Duke equation, using linear rectangular element, the problem will reduce to the following generalized eigenvalue problem.

$$
K u=\lambda M u
$$

Where matrices $K$ and $M$ are $n \times n$ matrices, $u$ is the vector of unknowns of dimensionality $n$, and $n$ is the number of nodes.

where

$$
\begin{aligned}
& K=\left[K_{\mathrm{l} j}^{\varphi}\right] \text { and } M=\left[M_{\mathrm{ij}}^{\ominus}\right], i=1,2, \ldots, n, j=1,2, \ldots, n \times \\
& K_{\mathrm{i} j}^{\varphi}=\int_{\Omega}\left[\frac{-\hbar^{2}}{2 m} \psi_{i}^{\varphi} \frac{\partial \psi_{j}^{\varphi}}{\partial r}+\frac{\hbar^{2}}{2} r \frac{\partial \psi_{i}^{\varphi}}{\partial r}\left(\frac{1}{m} \frac{\partial \psi_{j}^{\varphi}}{\partial r}\right)+\frac{\hbar^{2} L^{2}}{2 m r} \psi_{i}^{\varphi} \psi_{j}^{\varphi}+\frac{\hbar^{2}}{2} r \frac{\partial \psi_{i}^{\varphi}}{\partial z}\left(\frac{1}{m} \frac{\partial \psi_{j}^{\varphi}}{\partial z}\right)\right] d r d z \\
& M_{i j}^{\ominus}=\int_{\Omega}[V-E] r \psi_{i}^{\varphi} \psi_{j}^{\varphi} d r d z
\end{aligned}
$$


To determine the element coefficient matrices $K$ and $M$ in (19) and (20), consider the rectangular element show the Fig (2).

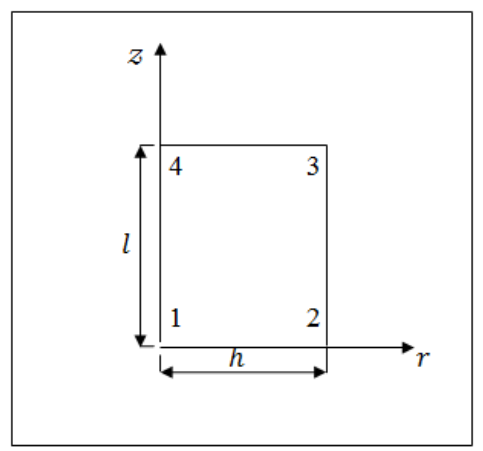

Fig.( 2) The rectangular element.

As a first choice the uniform mesh are used by four linear rectangular element, as shown in Fig. ( 3 ) to represent the domain (mesh Rect1), to determine the element coefficient matrices $K$ and $M$ of Eq. (18).

The elements 1, 2, 3, and 4 as shown in Fig.( 3) are identical in orientation as well as geometry. Then all four elements have the same element matrices, and it is necessary to compute them only for element 1 .

To evaluation of the integral in Eq. (19) and Eq. (20), it is possible to obtain the closed form for the $K$ and $M$ matrices by carrying out the integrals in Eq. (19) and Eq. (20) exactly respectively. We consider element 1 as the typical element. Hence, the element coefficient $K$ and $M$ matrices are

$$
\begin{aligned}
& {\left[K^{e}\right]=\frac{k_{e}}{2 h l}\left[\begin{array}{ccc}
h^{2}+l^{2} & -h^{2} & -l^{2} \\
-h^{2} & h^{2} & 0 \\
-l^{2} & 0 & l^{2}
\end{array}\right]} \\
& {\left[M^{e}\right]=\frac{k_{e 1}}{2 h l}\left[\begin{array}{ccc}
h^{2} l^{2}-2 h l^{2}-2 h^{2} l+h^{2}+2 h l+l^{2} & -h^{2} & -l^{2} \\
-h^{2} & h^{2} & 0 \\
-l^{2} & 0 & l^{2}
\end{array}\right]}
\end{aligned}
$$

Where $k_{e}=\frac{\hbar^{2}}{2 m} \frac{1}{12 A}$ and $k_{e 1}=[V-E] \frac{1}{12 A}$

The element matrices in Eq. (21) and Eq. (22) are valid for the Ben Daniel-Duke equation (4) on any right-angle triangle with sides $h$ and $l$.

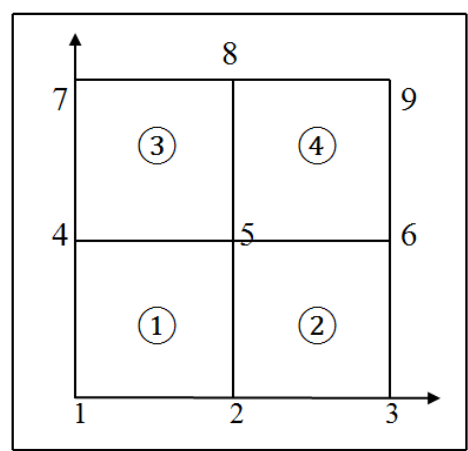

Fig.( 3) The uniform mesh Rect1 of four linear rectangular element.

Then the mesh shown in Fig. (3), will be as

$K=\left[\begin{array}{lll}A_{11} & A_{12} & A_{1 a} \\ A_{21} & A_{22} & A_{2 a} \\ A_{31} & A_{32} & A_{2 a}\end{array}\right]$

where

$$
\begin{aligned}
& A_{11}=\left[\begin{array}{ccc}
k_{11}^{1} & k_{12}^{1} & 0 \\
k_{21}^{1} & k_{22}^{1}+k_{11}^{2} & k_{12}^{2} \\
0 & k_{21}^{2} & k_{22}^{2}
\end{array}\right], A_{12}=\left[\begin{array}{ccc}
k_{14}^{1} & k_{13}^{1} & 0 \\
k_{24}^{1} & k_{2 a}^{1} & k_{13}^{2} \\
0 & k_{24}^{2} & k_{2 a}^{2}
\end{array}\right], A_{12}=\left[\begin{array}{ccc}
0 & 0 & 0 \\
0 & 0 & 0 \\
0 & 0 & 0
\end{array}\right], \\
& A_{21}=\left[\begin{array}{ccc}
k_{41}^{1} & k_{42}^{1} & 0 \\
k_{11}^{1} & k_{a 2}^{1}+k_{41}^{2} & k_{42}^{2} \\
0 & k_{a 1}^{2} & k_{a 2}^{2}
\end{array}\right], A_{22}=\left[\begin{array}{ccc}
k_{44}^{1}+k_{11}^{a} & k_{12}^{1} & 0 \\
k_{24}^{1}+k_{21}^{a} & k_{2 a}^{1}+k_{44}^{2}+k_{22}^{a}+k_{11}^{4} & k_{43}^{2}+k_{12}^{4} \\
0 & k_{a 4}^{2}+k_{21}^{4} & k_{a 3}^{2}+k_{22}^{4}
\end{array}\right],
\end{aligned}
$$




$$
\begin{aligned}
A_{2 \mathrm{a}} & =\left[\begin{array}{ccc}
k_{14}^{\mathrm{a}} & k_{1 \mathrm{a}}^{\mathrm{a}} & 0 \\
k_{24}^{\mathrm{a}} & k_{2 \mathrm{a}}^{\mathrm{a}}+k_{14}^{4} & k_{1 \mathrm{a}}^{4} \\
0 & k_{24}^{4} & k_{2 \mathrm{a}}^{4}
\end{array}\right], A_{21}=\left[\begin{array}{lll}
0 & 0 & 0 \\
0 & 0 & 0 \\
0 & 0 & 0
\end{array}\right], A_{22}=\left[\begin{array}{ccc}
k_{41}^{\mathrm{a}} & k_{42}^{\mathrm{a}} & 0 \\
k_{21}^{\mathrm{a}} & k_{22}^{\mathrm{a}}+k_{41}^{4} & k_{13}^{4} \\
0 & k_{21}^{4} & k_{22}^{4}
\end{array}\right], \\
A_{2 a} & =\left[\begin{array}{ccc}
k_{44}^{\mathrm{a}} & k_{4 \mathrm{a}}^{\mathrm{a}} & 0 \\
k_{24}^{\mathrm{a}} & k_{2 \mathrm{a}}^{\mathrm{a}}+k_{44}^{4} & k_{43}^{4} \\
0 & k_{24}^{4} & k_{2 \mathrm{a}}^{4}
\end{array}\right] .
\end{aligned}
$$

The above assembled coefficient matrix for finite element mesh is of order $9 \times 9$. In this case the sub matrices (the integrals) of $k_{i j}^{e}$ compute by an analytic method. The MATLAB program is used to solve the sub matrices (the integrals) of $k_{\mathrm{ij}}^{\mathrm{Q}}$.

It is important to mention that the elements of the above sub matrices (the integrals) of $k_{\mathrm{ij}}^{\mathrm{e}}$ are calculated by an analytic method

\section{Notes (1):}

In the following examples, the representative set of parameters were used for the calculations were as:

Radius $R=100 \AA, L_{w}=50 \AA, \quad L_{b}=50 \AA, \quad m_{w}=0.067 m_{0}, \quad m_{b}=0.0919 m_{0} \quad V_{0}=0.23 \mathrm{eV}$. These parameters correspond to a $\mathrm{GaAs} / A l_{0.3} \mathrm{Ga} a_{0.7} A s$ structure.

\section{Example (1):}

Consider the Nanowire superlattice model, with the two layers, at one end there is a well and at the other there is a barrier (asymmetrical).

In this example the linear rectangular element was used to solve Ben Daniel-Duke equation. Equation (18) is solved using MATLAB program at $n=1296$. The results are shown in Fig (4).

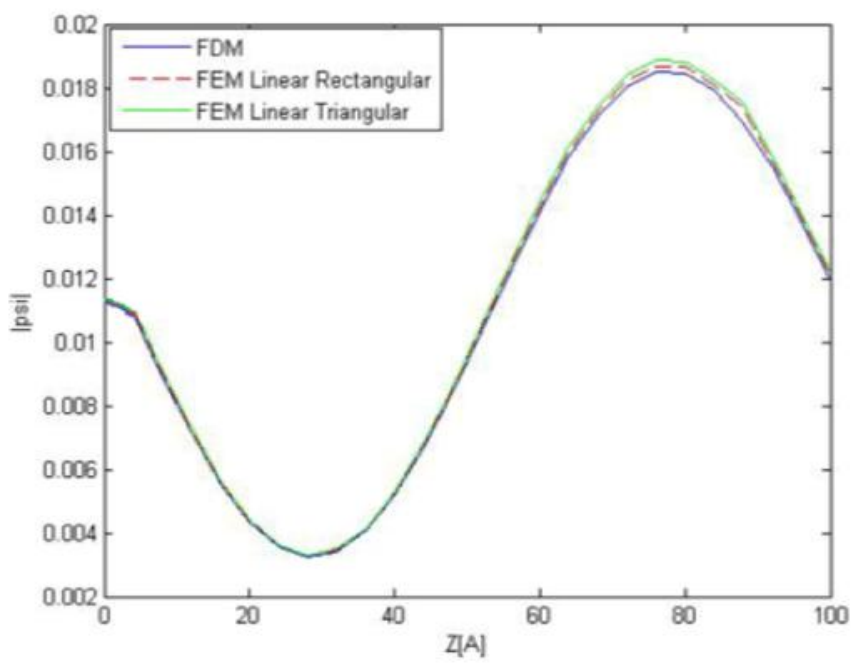

Fig. (4) Ground state for the NWSL calculated with the FEM of linear rectangular element, FEM of linear triangular element, and the FDM, discretization of the two layers, with at one end there is a well and at the other there is a barrier, asymmetrical.

\section{Example (2):}

Consider the Nanowire superlattice model for a three layers, with well layers at both ends (symmetrical).

In this example the linear rectangular element was used to solve Ben Daniel-Duke equation. Equation (18) is solved using MATLAB program at $n=1296$. The results are shown in Fig (5). 


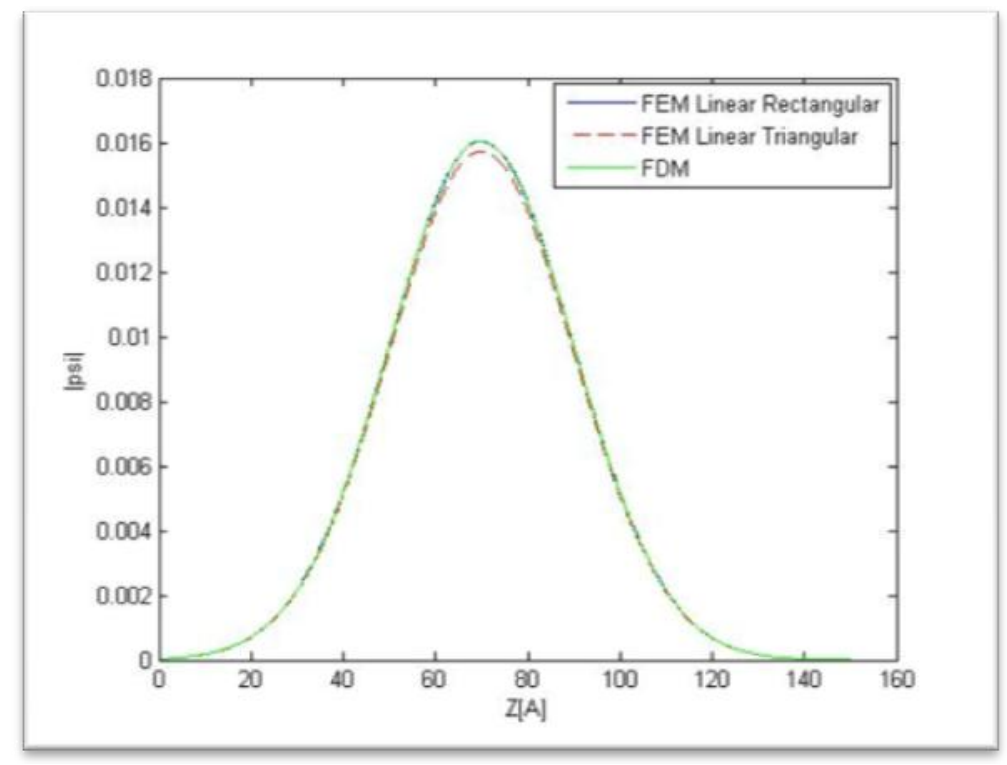

Fig. (5) Ground state for the NWSL calculated with the FEM of linear rectangular element, FEM of linear triangular element, and the FDM, discretization of the three layers, with well layers at both ends, symmetrical.

Example (3): Consider the Nanowire superlattice model for a six layers, at one end there is a well and at the other there is a barrier (asymmetrical).

In this example the linear rectangular element was used to solve Ben Daniel-Duke equation. Equation (18) is solved using MATLAB program at $n=1296$. The results are shown in Fig. (6).

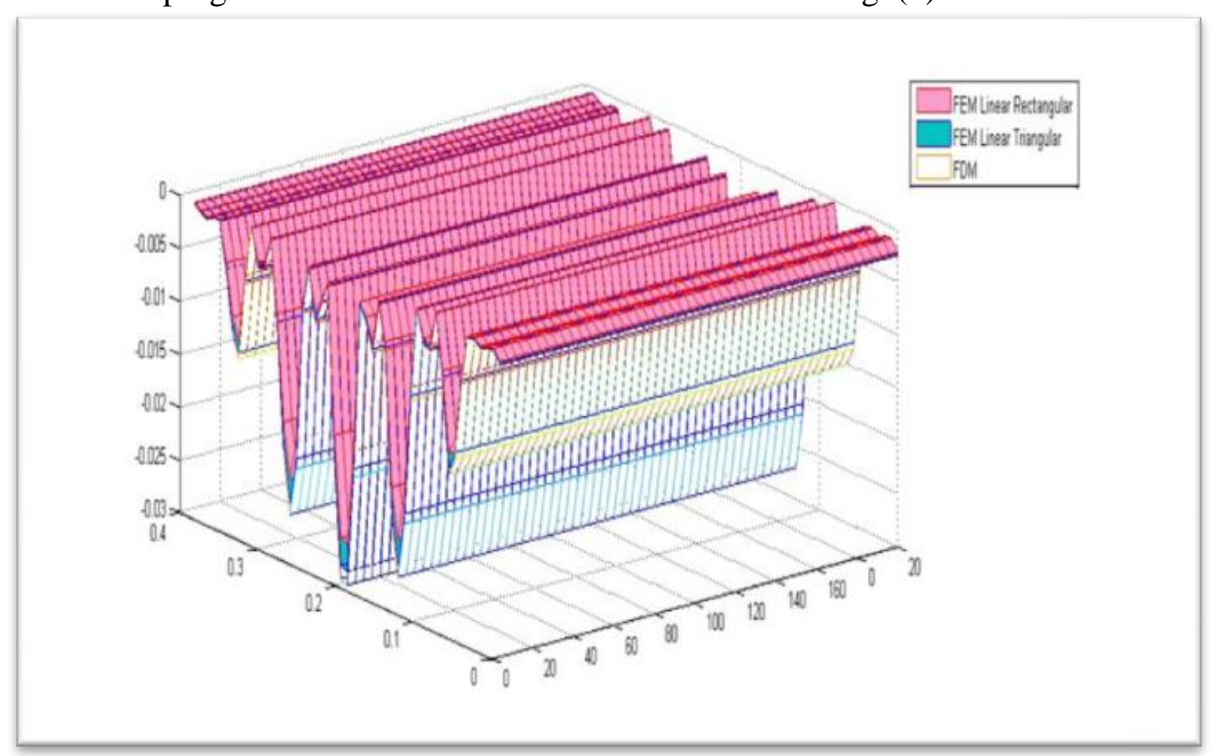

Fig. (6) Ground state for the NWSL calculated with the FEM of linear rectangular element, FEM of linear triangular element, and the FDM, discretization of the six layers asymmetrical.

\section{CONCLUSION}

The finite element method with using different base linear rectangular element are used for solving the one electron Ben Daniel-Duke equation with the solution of finite nanowire superlattice quantum dot structures GaAs/AlGaAs with a cylindrical cross-section and determine by electronic states in various type of layers in terms of wave functions between structures containing the same number of barriers and wells (asymmetrical) or containing a different number (symmetrical). The results of numerical examples are gives a good accuracy and efficiency of this method compare the results between finite difference methods and FEM of linear triangular element.

\section{REFERENCES}


[1]. D. J. BenDaniel and C. B. Duke ’space-charge effects on electron tunneling “' J. of Physical Review, Vol. 152, No. 2 (1966), pp. 683-692.

[2]. A. K. Ghatak, K. Thyagarajan and M. R. Shenoy ',A novel numerical technique for solving the one dimensional schroedinger equation using matrix approach - application to quantum well structures “ ' IEEE Journal of Quantum Electronics, Vol. 24, No. 8 (1988), pp. 1524-1531.

[3]. G. Paasch, P. H. Nguyen and G. Gobsch ''Envelope Equation and Wave Function Matching for Narrow-Gap Semiconductors“" Physica Status Solidi (b), Vol. 162, No. 1 (1990), pp. 155-163.

[4]. D. L. Mathine, S. K. Myjak and G. N. Maracas '’A Computational Fourier Series Solutin of the BenDaniel-Duke Hamiltonian for Arbitrary Shaped Quantum Wells“" IEEE Journal of Quantum Electronics, Vol. 31, (1995), pp. 1216-1222.

[5]. M. Willatzence, R. Melnik, C. Galeriu and L. C. Lew Yan Voon 'Finite Element Analysis of Nanowire Superlattice Structures“' Springer, Vol. 31, (2003), pp. 755-763.

[6]. C. Galeriu, L. C. Lew Yan Voon, R. Melnik and M. Willatzence ' Modeling a Nanowire Superlattice Using the Finite Difference Method in Cylindrical Polar Coordinates “ Computer Physics Communications, Vol. 157, (2004), pp. 147-159.

[7]. D. Sarkara, A. Deyasib 'Field Induced Tuning of DOS and Eigenstates in Double Quantum Well Structure having Gaussian Geometry“" American Scientific Publishers, Vol. 5, No. 1, (2016), pp. 138-143. 